\title{
Could Transgastric Endoscopic Ultrasound-Guided Aspiration Alone Be Effective for the Treatment of Pancreatic Abscesses?
}

\author{
Hoon-Gil Jo ${ }^{1}$, Baatarnum Amarbat ${ }^{2}$, Jin-Woo Jeong ${ }^{1}$, Hyo-Yeop Song', Seung-Ryel Song ${ }^{1}$ and Tae-Hyeon Kim ${ }^{1}$ \\ ${ }^{1}$ Department of Internal Medicine, Wonkwang University School of Medicine, Iksan, Korea, ${ }^{2}$ GI Endoscopy Center, National Cancer Center of \\ Mongolia, Ulaanbaatar, Mongolia
}

Drainage of pancreatic abscesses is required for effective control of sepsis. Endoscopic ultrasound (EUS)-guided endoscopic drainage is less invasive than surgery and prevents local complications related to percutaneous drainage. Endoscopic drainage with stent placement in the uncinate process of the pancreas is a technically difficult procedure. We report a case of pancreatic abscess treated by repeated EUS-guided aspiration and intravenous antibiotics without an indwelling drainage catheter or surgical intervention. Clin Endosc 2015;48:345-347

Key Words: Endoscopic drainage; Endoscopic ultrasound-guided fine needle aspiration; Pancreatic abscess

\section{INTRODUCTION}

A pancreatic abscess is an intra-abdominal collection of pus that occurs near the pancreas. It contains little or no pancreatic necrosis and results from acute pancreatitis, chronic pancreatitis, or pancreatic trauma. ${ }^{1}$ Pancreatic abscess is a serious medical condition that requires antibiotic therapy in conjunction with endoscopic, percutaneous, or surgical drainage. Endoscopic ultrasound (EUS)-guided endoscopic drainage is less invasive than surgery and does not require general anesthesia. ${ }^{2}$ Furthermore, recovery is faster, with lower morbidity and cost. Although EUS can be used to visualize excessive pancreatic fluid in the uncinate process from both the stomach and the duodenum, endoscopic drainage can be difficult to perform. We present what we believe to be the only reported case of a symptomatic abscess in the uncinate process of the pancreas to be successfully treated by transgastric EUS-guided aspiration without drainage catheter placement or surgical

Received: May 28, 2014 Accepted: July 4, 2014

Correspondence: Tae Hyeon Kim

Department of Internal Medicine, Wonkwang University School of Medicine, 895 Muwang-ro, Iksan 570-974, Korea

Tel: +82-63-850-2564, Fax: +82-63-855-2025, E-mail: kth@wonkwang.ac.kr

(cc) This is an Open Access article distributed under the terms of the Creative Commons Attribution Non-Commercial License (http://creativecommons.org/ licenses/by-nc/3.0) which permits unrestricted non-commercial use, distribution, and reproduction in any medium, provided the original work is properly cited. intervention.

\section{CASE REPORT}

A 51-year-old man presented to our hospital for abdominal pain and chills. He had noninsulin-dependent (type 2) diabetes mellitus, hypertension, and a history of pulmonary tuberculosis. He reported consuming two to three bottles of alcoholic beverages per day for the previous 20 years and had smoked one pack of cigarettes a day for the past 40 years. The patient's blood pressure was $90 / 50 \mathrm{~mm} \mathrm{Hg}$, pulse rate was 86 beats per minute, respiratory rate was 20 breaths per minute, and body temperature was $36.2^{\circ} \mathrm{C}$. On physical examination, the patient appeared acutely ill and was mentally alert. His abdomen was soft and flat, and bowel sounds were present. There was tenderness in the right upper quadrants. Blood levels of amylase, lipase, $\alpha$-fetoprotein, and carbohydrate antigen 19-9 were normal, and the results of liver function tests were within normal limits. Contrast-enhanced computed tomography (CECT) of the abdomen and pelvis revealed a fluid- and gas-containing lesion at the pancreatic head, indicative of a pancreatic abscess measuring $4.6 \times 3.6 \mathrm{~cm}$, without any other overt pancreatic parenchymal changes (Fig. 1).

For diagnosis and drainage of the pancreatic abscess, both radial EUS (GF-UE 260-AL5; Olympus Co., Tokyo, Japan) and 
linear (UCT240-AL5; Olympus Co.) echo-endoscopes were introduced into the duodenal bulb for a transduodenal approach to the pancreatic abscess. During EUS evaluations, an active, large, and deep ulcer with an exposed vessel on the duodenal bulb was revealed (Fig. 2). We initially planned a transduodenal approach to the pancreatic abscess for EUS-guided fine needle aspiration (FNA). Unfortunately, this active duodenal ulcer required that we change our plan. The EUS endoscope was instead introduced into the antrum of the stomach, and ultrasound showed a low echoic lesion with an irregular wall including floating material, measuring $3.6 \times 2.1 \mathrm{~cm}$ at the head of the pancreas (Fig. 3A). After selecting an appropriate aspiration site by ultrasound and Doppler inspection, a 22-gauge needle (EchoTip Ultra, ECHO 3-22; Cook Endoscopy, Winston-Salem, NC, USA) was introduced into the abscess (Fig. 3B) at the antrum; $14 \mathrm{~mL}$ of thick, purulent fluid with blood clots was aspirated. Prophylactic antibiotics had been administered on the day of the procedure. Cytological examination revealed inflammatory cells. Microbial analysis

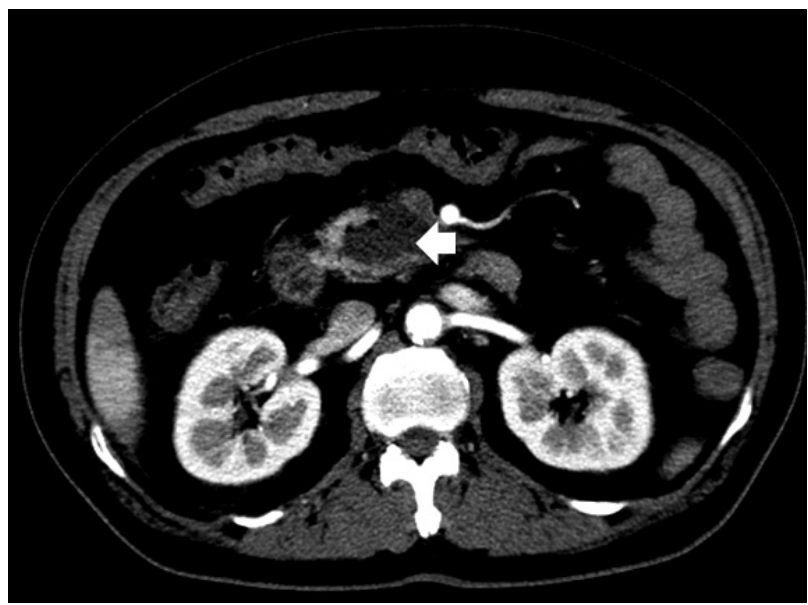

Fig. 1. Contrast-enhanced computed tomography. A gas-containing cystic lesion (arrow) suggestive of an abscess is visible in the pancreatic head portion (uncinate portion). of the purulent material showed Klebsiella pneumoniae. Based on the bacterial culture results, the patient was administered third-generation cephalosporins intravenously for 10 days. On the seventh admission day, fever and leukocytosis occurred; the pancreatic lesion was again aspirated with the intention of complete drainage of the cavity using EUS-FNA 22-gauge. A total of $7 \mathrm{~mL}$ of purulent fluid was obtained, and the cavity size decreased after this second aspiration. The patient had no immediate or late complications, and his clinical and laboratory findings improved. After 3 months, the follow-up CECT demonstrated successful resolution of the pancreatic abscess.

\section{DISCUSSION}

Drainage of pancreatic abscesses is required for the effective control of sepsis. The past two decades have seen an increase in the use of endoscopic management of symptomatic pancre-

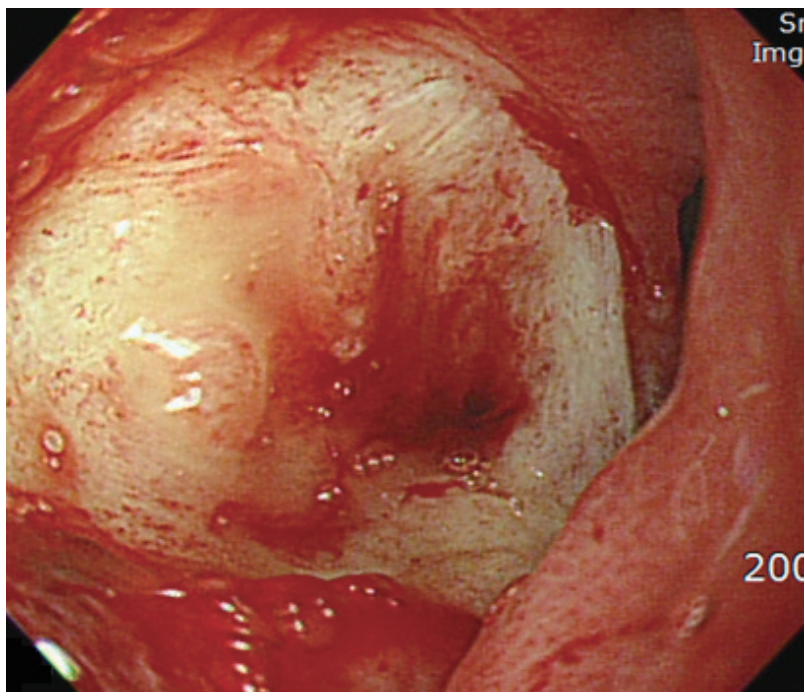

Fig. 2. Endoscopic finding. A large, ulcerated crater with exposed vessel is visible in the duodenal bulb.
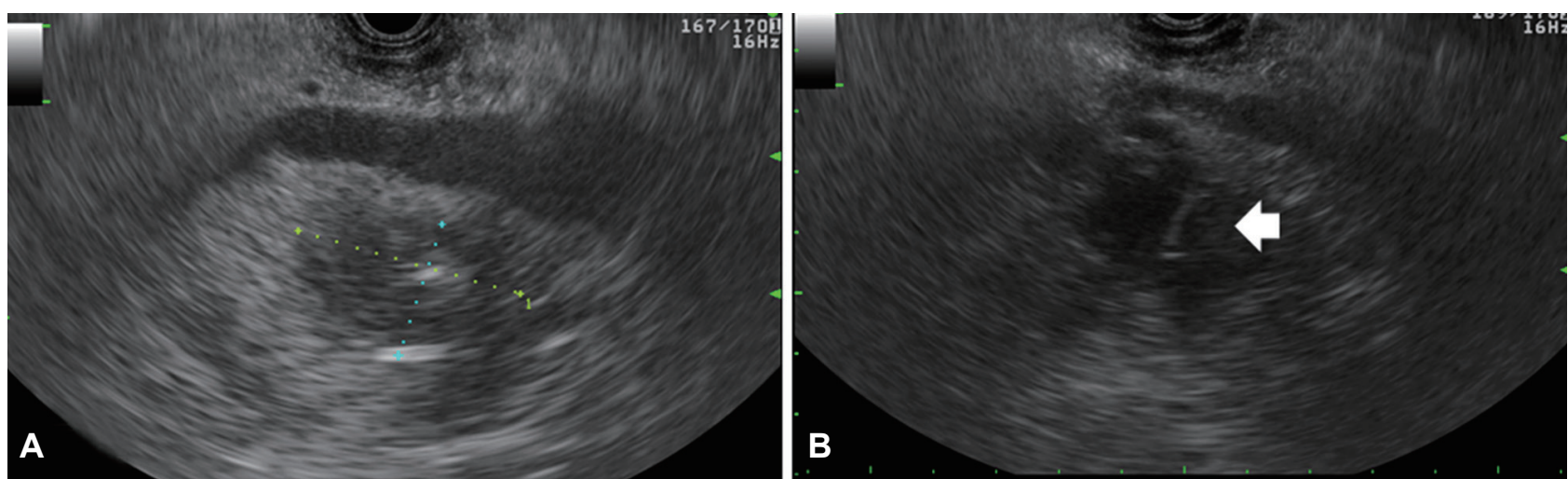

Fig. 3. Endoscopic ultrasound (EUS) findings (transgastric view). (A) A low echoic lesion with irregular wall measuring approximately $3.6 \times 2.1 \mathrm{~cm}$ is seen in the pancreatic head portion, suggestive of an abscess cavity. (B) A 22-gauge EUS-fine needle aspiration needle entering the cystic cavity for aspiration is visible (arrow) 
atic pseudocysts and abscesses. More recently, with the advent of EUS guidance and increasing experience, endoscopic management of more complex pancreatic fluid collections and nonbulging pancreatic pseudocysts has been feasible in selected patients, resulting in a reduced risk of procedure-related bleeding. ${ }^{3}$ Our patient with a pancreatic abscess involving the uncinate region was successfully treated with EUS-guided aspiration and intravenous antibiotic therapy alone, without placement of an indwelling drainage catheter or surgical intervention.

A prerequisite for EUS-guided drainage is the presence of a well-defined mature wall on ultrasound. The fluid collection must be endoscopically accessible; for example, it should be located within $1 \mathrm{~cm}$ of the duodenal or gastric walls, in order to avoid perforation and bleeding. For pancreatic abscess drainage, it may be necessary to dilate the transmural tract for drainage catheter insertion and stent placement. A great distance between the cavity and gastric or duodenal walls can lead to complications such as perforation and bleeding. Varadarajulu et al. ${ }^{4}$ reported that perforation was more common with pancreatic fluid collection in the uncinate region than in other locations. In this case, the patient had an abscess in the uncinate region of pancreas. Initially, we planned a transduodenal approach to the pancreatic abscess for EUS-guided drainage with catheter placement. However, the patient had a large, deep ulcer with an exposed vessel on the duodenal bulb, so a transgastric approach to the pancreatic head abscess was instead implemented for EUS-guided aspiration.

Uncinate masses in the pancreas are typically accessed transduodenally for EUS-FNA, and transgastrically if the lesion is large. For peri-pancreatic head lesions, when the endoscope is advanced to the duodenal bulb and the second portion, the endoscope is angulated and movement of the aspiration needle is too difficult for effective EUS-FNA in the working channel. ${ }^{5}$ The stiffness of 19-gauge FNA needles used for EUS-guided drainage of peri-pancreatic fluid collection can disturb this procedure in the duodenum. However, a transgastric approach to peri-pancreatic head lesions in the antrum or lower body of the stomach may be useful because the endoscope can be straightened and the FNA needle easily manipulated. In addition, large lesions in the uncinate region can be seen and sampled from the stomach, as in this case. The large distance between the fluid collection and the stomach made placement of drainage catheters difficult. However, aspiration via a transgastric EUS-FNA was possible. From a therapeutic standpoint, we can access a nonbulging abscess in the uncinate process of the pancreas via a transgastric route by linear EUS-FNA 22-gauge needle and aspirate the abscess without a drainage catheter or stent placement in the abscess cavity.

In conclusion, this was a case in which transgastric endoscopic EUS-guided aspiration was effective for the treatment of a pancreatic abscess. When placement of a naso-cystic catheter or plastic stent in a pancreatic abscess is possible, EUS-guided aspiration alone may be safer and more efficacious.

\section{Conflicts of Interest}

The authors have no financial conflicts of interest.

\section{Acknowledgments}

This paper was supported by 2013 research funds from Wonkwang University.

\section{REFERENCES}

1. Baillie J. Pancreatic pseudocysts (part I). Gastrointest Endosc 2004;59: 873-879.

2. Varadarajulu S, Lopes TL, Wilcox CM, Drelichman ER, Kilgore ML, Christein JD. EUS versus surgical cyst-gastrostomy for management of pancreatic pseudocysts. Gastrointest Endosc 2008;68:649-655.

3. Piraka C, Shah RJ, Fukami N, Chathadi KV, Chen YK. EUS-guided transesophageal, transgastric, and transcolonic drainage of intra-abdominal fluid collections and abscesses. Gastrointest Endosc 2009;70:786-792.

4. Varadarajulu S, Christein JD, Wilcox CM. Frequency of complications during EUS-guided drainage of pancreatic fluid collections in 148 consecutive patients. J Gastroenterol Hepatol 2011;26:1504-1508.

5. Jenssen C, Dietrich CF. Endoscopic ultrasound-guided fine-needle aspiration biopsy and trucut biopsy in gastroenterology: an overview. Best Pract Res Clin Gastroenterol 2009;23:743-759. 